\title{
Osteoporosis, CTCAE 5.0
}

National Cancer Institute

\section{Source}

National Cancer Institute. Osteoporosis, CT CAE 5.0. NCI Thesaurus. Code C146767.

A disorder characterized by reduced bone mass, with a decrease in cortical thickness and in the number and size of the trabeculae of cancellous bone (but normal chemical composition), resulting in increased fracture incidence. 